\title{
Studies on Chitin X. Cyanoethylation of Chitin
}

\author{
Seiichi TOKURA, Norio NISHI, Shin-ichiro NISHIMURA, \\ and Yoshihiro IKEUCHI \\ Department of Polymer Science, Faculty of Science, \\ Hokkaido University, Sapporo 060, Japan
}

(Received April 4, 1983)

\author{
KEY WORDS Cyanoethyl-Chitin \\ Acrylonitrile / Solubility / Chitin /
}

IR Spectra / Alkali-Chitin /
Chitin is one of the inert polysaccharides which supports the curticles of Crustacea and known to be present abundantly in nature. Its chemical modifications, such as acylations ${ }^{1-4}$ and alkylations, ${ }^{5,6}$ have been studied at our laboratory so as to utilize it effectively as a natural resource. In the course of these studies, chitin was found to be converted successfully to water-soluble materials by carboxymethylation or dihydroxypropylation. ${ }^{6}$ The hydrophilicity of chitin was also enhanced remarkably by the alkylation reaction with hydrophobic alkyl groups having linear or branched chains, in spite of the relatively low degree of substitution. ${ }^{5}$ The solubility was also enhanced toward general organic solvents by acylation reactions. ${ }^{1-4}$ The initial alkylation site was assumed to be the $\mathrm{C}_{6}$ hydroxy group in the $\mathrm{N}$-acetylglucosamine residue by ${ }^{13} \mathrm{C}$ NMR study. ${ }^{5}$

The cyanoethylation of chitin seems to be of interest from the standpoint of such solubility increment especially in organic solvents. Further, cyanoethyl-chitin seems to be a very important material since it can be an intermediate for further chemical modifications. However, the cyanoethylation of chitin must be achieved under rather milder conditions than that of cellulose ${ }^{7}$ owing to the unstable acetamide group at the $\mathrm{C}_{2}$ position. Therefore, the cyanoethylation reaction was carried out in a limited amount of alkali at a rather lower temperature. The results on the cyanoethylation of chitin are reported in brief in this paper.

\section{EXPERIMENTAL}

\section{Materials}

Chitin was prepared from Queen Crab shells according to the method of Hackman ${ }^{8}$ and powdered to 45-60 mesh before use. Acrylonitrile was purchased from Wako Pure Chemical Industries, Ltd. and purified by being passed through a silica gel column (Nakarai Chemicals Ltd., silica gel No. $\mathrm{V}, 70-325$ mesh). Deuterium oxide $\left(\mathrm{D}_{2} \mathrm{O}\right.$, above $99.85 \%$ ) was obtained from Commissariat à l'Énergie Atomique (CEA) of France. Other reagents of reagent grade were obtained from Wako Pure Chemical Industries, Ltd. and used without further purification.

\section{Preparation of Alkali-Chitin}

$10 \mathrm{~g}$ of chitin powder was suspended in a freshly prepared sodium hydroxide solution $(40 \mathrm{ml})$ of various concentrations including $0.2 \% \mathrm{SDS}$, and the mixture was treated similarly as reported previously. ${ }^{5}$

\section{Cyanoethylation of Chitin}

Frozen alkali-chitin was suspended directly in acrylonitrile or an acrylonitrile-organic solvent mixture. The mixture was made to stand at 12$14^{\circ} \mathrm{C}$ for $18 \mathrm{~h}$ with occasional stirring and then neutralized with diluted aqueous acetic acid at an ice-cold temperature. The precipitate was collected by filtration and washed successively with ethanol, DMF, ethanol, and acetone. The air-dried crude product was washed with DMF under reflux in a 
Soxhlet apparatus and air-dried again after successive washing with DMF and ethanol.

The DMF extracts were combined and DMF was evaporated off in vacuo. The residue was washed with acetone and air-dried.

\section{$\mathrm{D}_{2} \mathrm{O}$ Treatment of Chitin and Chitin Derivatives}

Powders of chitin and chitin derivatives were treated with $\mathrm{D}_{2} \mathrm{O}$ for $12 \mathrm{~h}$ at room temperature and then air-dried. The DMF-soluble portion of cyanoethyl-chitin was redissolved in DMF and cast on a glass plate. DMF was evaporated off on a hot plate. The resulting films were treated with $\mathrm{D}_{2} \mathrm{O}$ similarly as the powdered samples. Chitin and chitosan films ${ }^{9,10}$ were also treated with $\mathrm{D}_{2} \mathrm{O}$ similarly.

\section{Infrared Absorption Spectra}

IR spectra were measured as $\mathrm{KBr}$ pellets for the powdered samples or directly for the film samples using a JEOL A-302 spectrophotometer.

\section{Degree of Cyanoethylation}

The degree of cyanoethylation was estimated by nitrogen analysis using Coleman Nitrogen Analyzer, Model 29 on the basis that the Nacetylglucosamine residue contains $1 / 2 \mathrm{~mol}$ of adsorbed water up to 1.0 substitution as reported previously. ${ }^{1}$

\section{RESULTS AND DISCUSSION}

As the cyanoethyl group is introduced into the $\mathrm{N}$ acetylglucosamine residue under a strongly alkaline condition, polyacrylonitrile may possibly be formed as a by-product during the substitution reaction. Thus, the successive washing process with hot DMF is essential to remove the homopolymer.

The oxidation of cyano groups and dissociation of cyanoethyl groups from cellulose were reported to occur in the cyanoethylation reaction of cellulose due to a high alkaline concentration. ${ }^{11}$ Oxidation of the cyano group was suggested also in the cyanoethylation of chitin by the IR spectrum, when the alkaline concentration was very high. The deacetylation reaction was also observed in these cases. The relationship between the degree of substitution as estimated from the nitrogen analysis and the alkaline concentration in the reaction mixture is shown in Figure 1. The molar ratio of alkali

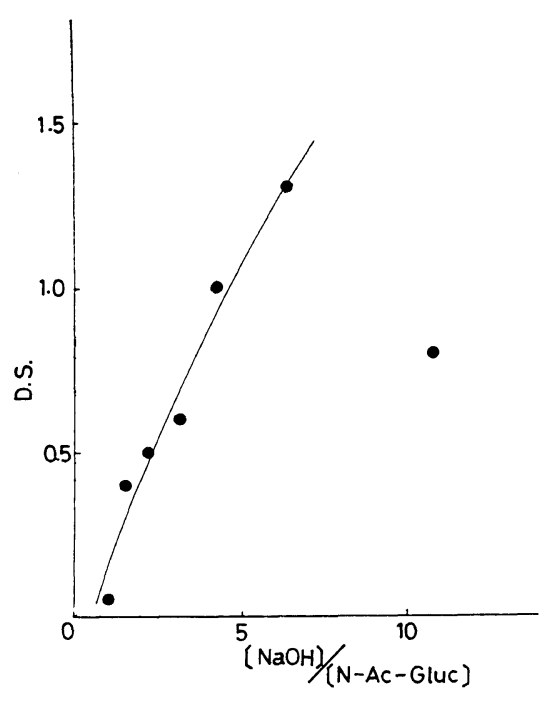

Figure 1. Relationship between the degree of substitution (D.S.) and the amount of $\mathrm{NaOH}$ employed in the cyanoethylation reaction.

to the $N$-acetylglucosamine residue seems to be quite essential to prepare cyanoethyl-chitin without oxidation or deacetylation reaction, since the degree of substitution tended to reduce remarkably at a higher molar ratio than 10 . The highest degree of substitution was given at the molar ratio around 7 under our experimental conditions.

Several IR spectra of variously cyanoethylated chitins are shown in Figure 2 along with that of the DMF-soluble fraction. Increase in the degree of cyanoethylation is clearly shown by the increase in absorptions at $2400 \mathrm{~cm}^{-1}$ due to cyano groups and at $2880 \mathrm{~cm}^{-1}$ due to stretching of methylene groups. Though there are two absorption peaks at the amide I region in native chitin ( $\beta$-chitin), ${ }^{12}$ an absorption at $1620 \mathrm{~cm}^{-1}$ dissappears with the progress of cyanoethylation as shown in Figure 2, and there is no absorption peak at $1620 \mathrm{~cm}^{-1}$ in 1.0 cyanoethylated chitin. ${ }^{13} \mathrm{C}$ NMR studies of the cyanoethylchitin in deuterated formic acid revealed that the $\mathrm{C}_{6}$ hydroxyl group of the $\mathrm{N}$-acetylglucosamine residue was the initial cyanoethylation site as in the case of other alkylation reactions. ${ }^{5}$ As the absorption at $3350 \mathrm{~cm}^{-1}$ due to water was not seen in highly substituted cyanoethyl-chitin, the absorption at $1620 \mathrm{~cm}^{-1}$ of chitin seems to be due to the hydrogen bond between the $\mathrm{C}_{6}$ hydroxyl group and the acetamide group at the $\mathrm{C}_{2}$ position. This hydrogen 


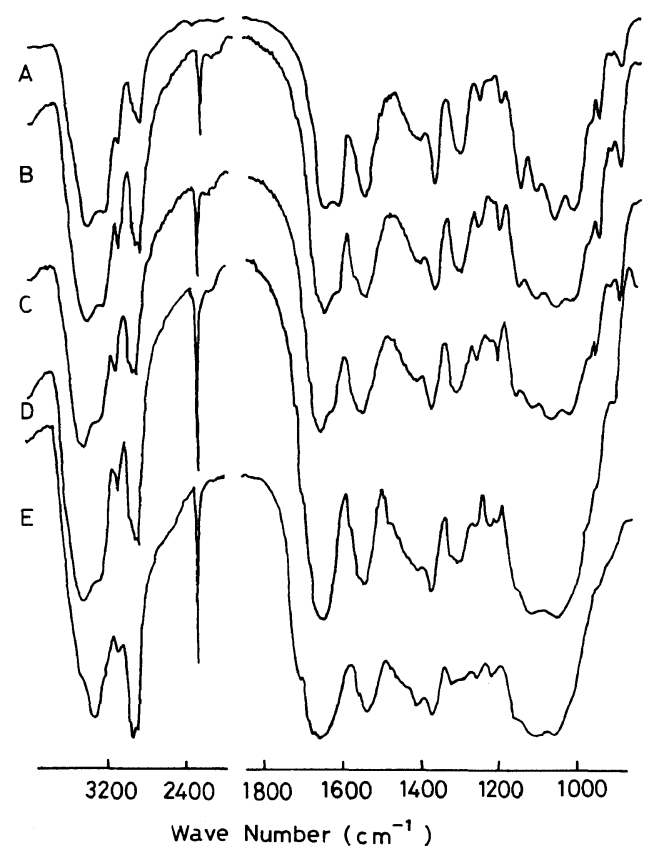

Figure 2. Infrared absorption spectra of (A) chitin, (B) 0.4 cyanoethyl-chitin, (C) 0.6 cyanoethyl-chitin, (D) 1.0 cyanoethyl-chitin, and (E) a DMF-soluble part from 0.6 cyanoethyl-chitin.

bond is likely formed through a water molecule. The absorption at $1060 \mathrm{~cm}^{-1}$ due to the primary hydroxyl group also decreased by the progress of cyanoethylation. The IR spectrum of a DMFsoluble part from 0.6 cyanoethylated chitin suggests the presence of cyanoethyl-chitin in the DMF extract. However, as the contamination with polyacrylonitrile was also assumed from the IR spectrum further fractionation should be performed for this DMF-soluble part.

Chitin and the chitin derivatives were treated with $\mathrm{D}_{2} \mathrm{O}$ and their IR spectra were measured in order to obtain in understanding of the contribution of water molecules to the formation of rigid crystalline structure in natural chitin. Several peaks due to the adsorbed $\mathrm{D}_{2} \mathrm{O}$ were observed in the IR spectra as shown in Figure 3 and could be divided into two groups, one around $2600 \mathrm{~cm}^{-1}$ and the other around $2500 \mathrm{~cm}^{-1}$. The former absorption seems to depend on the rigid crystalline structure of chitin molecules, since this peak tends to reduce by mercerization or the progress of cyanoethylation. The IR spectrum of the $\mathrm{D}_{2} \mathrm{O}$-treated regenerated chitin film

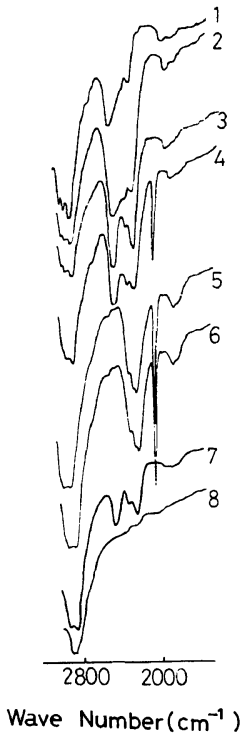

Figure 3. Infrared absorption spectra of $\mathrm{D}_{2} \mathrm{O}$-treated chitin and chitin derivatives: 1 , chitin powder; $2, \mathrm{NaOH}$ treated chitin; $3,0.05$ cyanoethyl-chitin; 4, 0.4 cyanoethyl-chitin; $5,0.6$ cyanoethyl-chitin; $6,1.0$ cyanoethyl-chitin; 7 , chitin film ${ }^{10} ; 8$, chitosan film. ${ }^{9}$

prepared from a formic acid solution suggests that about $60 \%$ of the crystalline structure is reformed compared to that of the native chitin powder. The absorption of $\mathrm{D}_{2} \mathrm{O}$ was hardly observed in the chitosan film, ${ }^{9}$ which was a deacetylated product of chitin. The acetamide group at the $\mathrm{C}_{2}$ position of the $N$-acetylglucosamine residue may be essential to form the rigid crystalline structure of chitin. On the other hand, the absorptions around $2500 \mathrm{~cm}^{-1}$, which increase with an increase in the degree of cyanoethylation, may be attributed to the effect of $\mathrm{D}_{2} \mathrm{O}$ on the amorphous part of the molecule. However, as a clear interpretation can not be made so far, further investigation is necessary to clarify this phenomenon.

As described above, the solubility of cyanoethylchitin enhanced remarkably especially in formic acid, and the preparation of its fiber or film was found to be much easier than that of chitin. ${ }^{10}$ The solubility of cyanoethyl-chitin seems to increase with an increase in the degree of substitution. Preparation, characterization, and utilization of its fiber and film will be discussed together with a more detailed conformational investigation in a future paper. 


\section{S. TOKURA et al.}

Acknowledgement. The authors should like to express their appreciation to Dr. Akihiro Tsutsumi of our department for his valuable comments on the NMR study.

\section{REFERENCES}

1. N. Nishi, J. Noguchi, S. Tokura, and H. Shiota, Polym. J., 11, 27 (1979).

2. O. Somorin, N. Nishi, S. Tokura, and J. Noguchi, Polym. J., 11, 391 (1979).

3. K. Kaifu, N. Nishi, T. Komai, S. Tokura, and O. Somorin, Polym. J., 13, 241 (1981).

4. N. Nishi, H. Ohnuma, S. Nishimura, O. Somorin, and S. Tokura, Polym. J., 14, 919 (1982).

5. S. Tokura, J. Yoshida, N. Nishi, and T. Hiraoki, Polym. J., 14, 527 (1982).

6. S. Tokura, N. Nishi, A. Tsutsumi, and O. Somorin, Polym. J., in press.

7. M. Negishi, I. Sekiguchi, and S. Okada, Sen- $i$ Gakkaishi, 12, 479 (1956).

8. R. H. Hackman, Aust. J. Biol. Sci., 7, 168 (1958).

9. S.Tokura et al., unpublished data.

10. S. Tokura, N. Nishi, and J. Noguchi, Polym. J., 11, 781 (1979).

11. J. W. Frick, W. A. Reeves, and J. D. Gutherie, Text. Res. J., 27, 92 (1957).

12. F. G. Pearson, R. H. Marchessault, and C. Y. Liang, J. Polym. Sci., 18, 101 (1960). 